\title{
Influenza, HIV, Coronavirus: Limited/Incomplete Sustained Response to Therapy?
}

\author{
Daniel Amsterdam PhD* \\ Department of Microbiology \& Immunology, Medicine and Pathology, Jacobs School of Medicine and Biomedical Sciences, Buffalo NY, USA
}

${ }^{*}$ Corresponding Author: Daniel Amsterdam PhD, Department of Microbiology \& Immunology, Medicine and Pathology, Jacobs School of Medicine and Biomedical Sciences, Buffalo NY, USA; E-mail: amsterda@buffalo.edu

Received: March 09, 2020; Accepted: March 12, 2020; Published: March 14, 2020

The three viruses addressed in this opinion/review have in common that their genetic material is RNA and is single stranded. Viral taxonomists do not consider HIV an RNA virus, as it has a DNA intermediate in its replication cycle and it is therefore referred to as a retrovirus. Generally, RNA viruses have exceedingly high mutation rates compared to DNA viruses because the enzyme required for polymerization - RNA polymerase — does not possess the proofreading capability of DNA polymerase. Because of this fallibility it is difficult to make effective vaccines for diseases caused by RNA viruses. Retroviruses, in specific HIV, possess a high mutation rate even though their DNA intermediate is subject to host DNA proofreading as errors during reverse transcription are associated with both strands of DNA before integration.

For each of these viral entities, transmission through an intermediate animal host has been required to develop human disease. In the case of influenza - bird or pig; for HIV - monkey; and for the newest COVID agent (SARS-CoV-2) a civet. For influenza, annual vaccines (sometimes biannual) are available based upon the previous year's activity. Their effectiveness varies from year to year and recipient age, ranging between $40-60 \%$. Typically, inactivated influenza vaccines rely on antibody response to achieve protection, while DNA vaccines can effectively interact with humoral and cell-mediated responses. DNA vaccines for influenza have been in design since the 1990's but have been slow in development. An advantage of DNA vaccines is that they forego the necessity of live virus reproduction and can be rapidly designed and upscaled to meet ever-changing seasonal variations. More contemporary seasonal vaccines provide little to no protection against novel pandemic viruses of animal origin.

In the case of individuals who have not been vaccinated against influenza there are four FDA-approved anti-viral drugs recommended by the Centers for Disease Control (CDC). These antivirals work best when taken within 48 hours of onset of illness to reduce the duration of infection and potentially prevent severe flu complications.

For HIV there are several FDA-cleared anti-retroviral agents (ARTs) and these have the capability to markedly reduce the viral load of infected individuals and extend the life of AIDS patients but they do not eliminate the virus. HIV can persist at undetectable levels in blood and cryptic tissue sites of infected patients. Although ARTs were developed early in the investigation of the disease, vaccines were considered the path to follow for eliminating AIDS. Yet, after more than $30+$ years, no vaccine has been developed and trialed with acceptable performance to warrant marketing. In fact, the HIV vaccine study known as "HVTN 702" which began in October 2016 and was expected to show hints of working was stopped early for absence of efficacy.

For the recently diagnosed severe acute respiratory syndrome labelled COVID-19 caused by coronavirus-2 (SARS-CoV-2) it is too early to reference any effective anti-infective agent or vaccine. In fact the problem has been complicated by diagnosis and diagnostics - that is a limited supply of viral test kits which are based on the polymerase chain reaction (PCR) to identify individuals with the disease. Synthetic antibody tests are also in development. These will do little to diagnose individuals with the disease, i.e. acute cases, but will help clarify questions about the spread of the infection and become an inventory for reagents in vaccine development.

Although the genetic sequence of the virus is known, it will take at least one year to design and trial an effective vaccine and several pharmaceutical companies are working to develop and deliver a vaccine to NIH for early testing. Currently there is no approved antiviral agent for SARS - CoV-2, although the nucleotide analog, remdesivir has shown antiviral activity against other coronaviruses, namely MERS and SARS.

Clearly, the near universal and lifelong protection that vaccines have achieved with bacterial infections is absent with the three viral agents discussed here. It will require additional research initiatives and monetary investment to reach that goal.

Citation:

Daniel Amsterdam PhD (2020) Influenza, HIV, Coronavirus: Limited/ Incomplete Sustained Response to Therapy? Microbiol Immunol Pathol, Volume 1(1): 1-1. 